\title{
Long-Term Measurement of Maternal Pulse Rate Dynamics Using a Home-Based Sleep Monitoring System
}

\author{
Ying Chen, ${ }^{1}$ Wenxi Chen, ${ }^{1}$ Kei-ichiro Kitamura, ${ }^{2}$ and Tetsu Nemoto ${ }^{2}$ \\ ${ }^{1}$ Biomedical Information Technology Laboratory, The University of Aizu, Ikki-machi, Aizuwakamatsu, Fukushima 965-8580, Japan \\ ${ }^{2}$ Department of Laboratory Science, Institute of Medical, Pharmaceutical and Health Sciences, Kanazawa University, Kanazawa, \\ Ishikawa 920-1192, Japan
}

Correspondence should be addressed to Wenxi Chen; wenxi@u-aizu.ac.jp

Received 13 May 2015; Accepted 24 August 2015

Academic Editor: Guiyun Tian

Copyright (C) 2016 Ying Chen et al. This is an open access article distributed under the Creative Commons Attribution License, which permits unrestricted use, distribution, and reproduction in any medium, provided the original work is properly cited.

\begin{abstract}
Major adaptations occur in the maternal cardiovascular system during pregnancy and after delivery; knowledge of these changes is essential to the health management of pregnant women. This paper presents a longitudinal study and proposes a methodology to monitor daily pulse rates (PRs) and characterize the weekly changes in maternal PRs before and after delivery. PRs were recorded during nightly sleep using an automatic monitoring system. PRs of the nonpregnant woman were taken as a reference. Weekly PR properties were characterized by histogram and a two-Gaussian mixture model. A coupling use of sample entropy and pulse rate was applied to cluster the stages during recovery period after delivery. Results depicted a profile of individual's cardiac recovery process in late pregnancy and after delivery. The results reveal that maternal PRs show different patterns in various stages of recovery. Later stages of recovery seemed to require a much longer period. The present study introduced a convenient approach in long-term maternal cardiac condition monitoring.
\end{abstract}

\section{Introduction}

The maternal cardiovascular system undergoes remarkable changes during pregnancy before and after delivery to meet the increased metabolic demands of pregnancy [15]. Recent laboratory studies have reported some specific turning points during restoration when significant changes of cardiac dynamics can be observed [1-3, 6-9]. However, so far, the extent and the timing of cardiac recovery have been a subject of debate. Heart rate (HR) was reported to return slowly to baseline levels by $2-6$ weeks postpartum in some studies [1, 2, 6, 9-11]. In other studies, a continued decrease in cardiac output was observed to last over the next 24 weeks [9]. However, there has also been concern that the changes in cardiac function associated with pregnancy might not ultimately return to prepregnancy levels $[8,9,11]$. The controversy was probably caused by different cohort and protocols used in data collection. Apart from cardiac adaptation, postpartum recovery can be influenced by many other factors, such as physical and age differences, mode of delivery, sufficient rest, appropriate exercise, and ample supplies of nutrients. Besides, if sympathetic-predominant autonomic balance is not smoothly recovered, postpartum women might become vulnerable to external stressors and may develop mental disorders which can inversely postpone the cardiac recovery [12-14].

Previous studies on maternal HR were mostly based on short durations [8, 15-17]. Few longitudinal analyses were performed to assess continuous HR changes individually during pregnancy. Even less information is available on how these changes in the HR develop during the postpartum period. The knowledge of these changes is essential to the management of women with cardiovascular disease and also helpful in health monitoring during and after normal pregnancy $[7,8,18,19]$.

Conventional noninvasive techniques of electrocardiogram (ECG), echocardiography, and echo Doppler methods can be used for the measurement of maternal cardiac activity without risk or discomfort to the subject. However, they are not suitable for daily application over a long-term period because of high cost and complexity. In the current study, we conducted a long-term observation of maternal pulse rate 


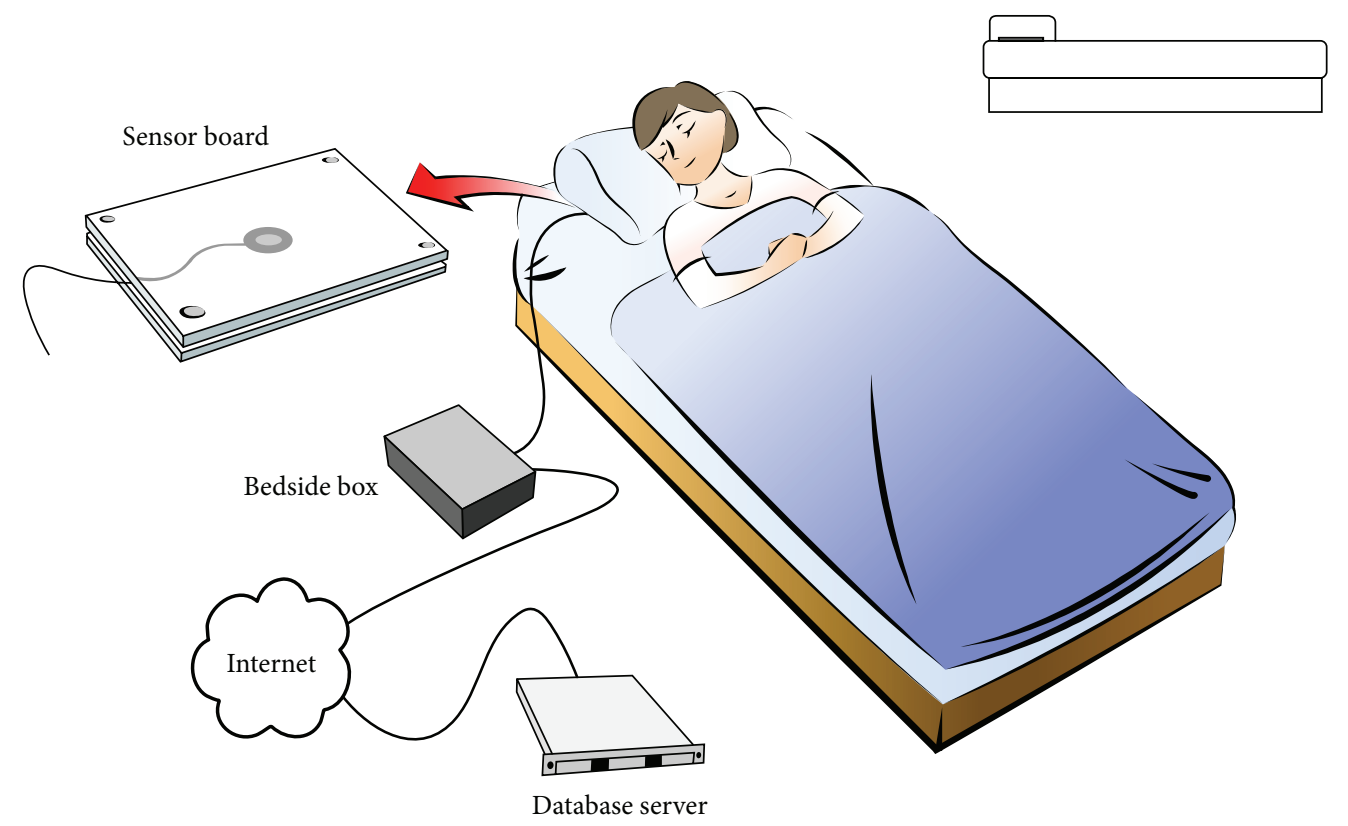

FIGURE 1: Experiment setting for long-term automatic data collection during sleep.

(PR) using a simple and low-cost sleep monitoring system during late pregnancy and up to 1 year after delivery. Pulse signal has been found as effective as ECG in measuring the parameters of heart rate variability (HRV) [20], widely applied for clinical physiological measurements [21-23], but its application for maternal cardiology during pregnancy is still rarely reported up to date. In this paper, the combined use of histogram and a two-Gaussian mixture model was applied to characterize weekly PR property. A coupling of nonlinear dynamical analysis by sample entropy (SampEn) and PR was used to classify stages after delivery. Some novel information about maternal cardiac recovery can be revealed by the introduced model and the combination of the features. This study serves to introduce a convenient protocol in daily PR monitoring and explore the potential applications of daily $\mathrm{PR}$ in revealing the progression of cardiac functions during pregnancy and its restoration after parturition.

\section{Materials and Methods}

2.1. Subjects. A 30-year-old healthy nulliparous woman (BMI before pregnancy: 21.2) with a singleton pregnancy was enrolled in this study. One year of PR data from a healthy nonpregnant female volunteer (BMI: 20.0) in her thirties was studied for reference. She did not have a history of pregnancy. Both subjects were free of any cardiac pathology or smoking or drinking habits or suffered from anxiety. The pregnant woman was in the absence of pregnancy induced hypertension and did not take any medicine during the recording period. She had a vaginal delivery and chose a mixed feeding (breast and bottle) after delivery. From 35th week postpartum, the baby had not lived with the mother. Informed consents were obtained from both subjects.

2.2. Experiment Setting and Data Collection. Both PR recordings were performed at each volunteer's home during their nightly sleep. Pulse signal was acquired by our own designed sleep monitoring system as shown in Figure 1, and the details of the system had been described in previous publications $[24,25]$. In order to facilitate usability in daily data collection, the system was developed based on Internet for daily measurement which causes neither restriction nor discomfort and brings no intervention to users' daily life. It can send the data automatically to a database server through Internet without any operations by the user so that a high rate in daily data collection can be achieved over long term. This system consisted of a sensor board, a bedside box, and a network database server. The sensor board was installed under the subject's pillow to measure the occipital pressure signals caused by heartbeats and respiratory movements. The bedside box amplified the analog pressure signal, digitized the signal at a sampling rate of $100 \mathrm{~Hz}$, and transmitted the data stream continuously to a database server via an Internet connection. This monitoring system was always in standby state. It was switched on $30 \mathrm{~s}$ after the subject lay down in the bed and was terminated and went back into standby mode $5 \mathrm{~min}$ after the subject rose in the morning.

Digital signal processing for pulse wave peak detection was implemented using a wavelet-based algorithm [26]. Figure 2 shows a sample of a raw pressure signal and the corresponding reconstructed pulse waveform. The pulse interval (PI) series was obtained as the sequence of the times occurring between each pair of consecutive pulse wave peaks. The corresponding PR (with unit of "beat per minute") can be computed by $\mathrm{PR}=1$ (minute) $\times 60(\mathrm{~s}) \times 1000$ $(\mathrm{ms}) / \mathrm{PI}$ (in $\mathrm{ms})=60000 / \mathrm{PI}$ (beat per minute). Performance of PR detection from the pressure signal was evaluated on the beat-by-beat basis using manual method by visually comparing with detections from finger photoplethysmogram measured simultaneously. The sensitivity and positive predictivity of PR detection were $98.91 \%$ and $98.47 \%$, respectively [26]. 


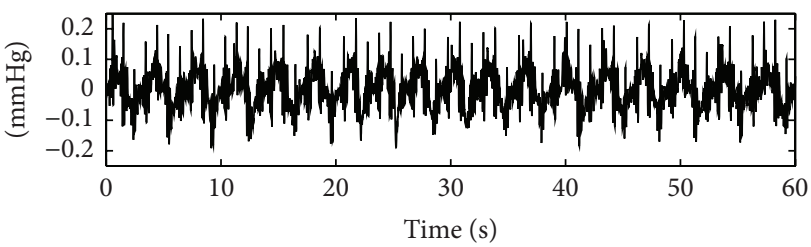

(a)

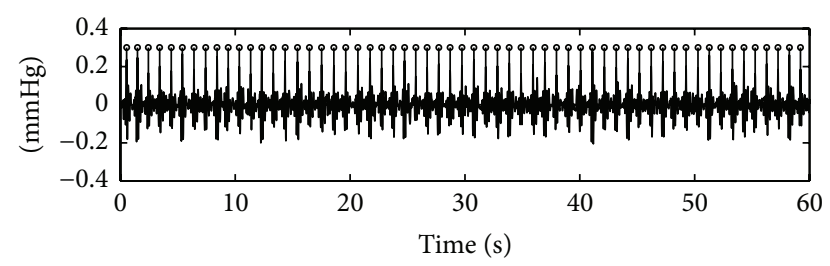

(b)

Figure 2: An example of fully detected beat-by-beat pulse rate in one minute from the pregnant woman. (a) The raw pressure signal, (b) the reconstructed pulse-related waveform. Open circles "o" indicate the detected characteristic points for pulse rate determination.

Data recording from the pregnant woman started from the late trimester (in the 30th week after conception, July 1 , 2010) and ended on the 63rd week postpartum (November 30 , 2011). Delivery occurred in the 40th week plus 2 days of gestation (September 14, 2010). A total of 373 nights of data were available for analysis. The woman stayed in the hospital for the first week after delivery; so the data was not recorded during that period. From the 2 nd week to the 6 th week, she returned home but did not sleep regularly in her original bed for taking care of the baby in another bed. Only sporadic data were collected during that period. A second lost period was from the 26th week to the 34th week postpartum because the subject was absent. Other sporadic data losses were because the subject was away from home. In the nonpregnant control individual, PR data were taken from April 1, 2004, to April 1, 2005; data were available for a total of 332 nights. Only PR data segments measured from 01:00 am to 07:00 am were used in this analysis.

2.3. Weekly Pulse Rate Modeling. We applied histograms graphically to represent the distribution of weekly PR data. A two-Gaussian mixture model was used to fit each weekly histogram to detect its two modes. Since weekly PR distributions may present different patterns throughout the recording period, they might be unimodal (single peak), flat (no peak), or bimodal (two distinct peaks). Different patterns might indicate different stages of PR development during pregnancy and recovery. Thus, a mixture model can better describe each distribution and help in understanding PR development and locating the transitions points of PR changes. The Gaussian model can be described by the following formula:

$$
f(x)=\sum_{i=1}^{2} a_{i} e^{-\left(x-b_{i}\right)^{2} / c_{i}^{2}}, \quad i=1,2,
$$

where $a_{i}$ was the peak value of the $i$ th model, indicating the percentage of the PR mean value in the $i$ th Gaussian component, $b_{i}$ was the peak position of the $i$ th model referring to the mean value of $\mathrm{PR}$ in the $i$ th Gaussian component, and $c_{i}$ was related to the standard deviation (SD) of the PR data series fitted by the $i$ th model. All the signal processing and data analysis were implemented using MATLAB and its "Curve Fitting Toolbox."

2.4. Sample Entropy. Sample entropy (SampEn), a refinement of the approximate entropy (ApEn) of statistics introduced by
Pincus [27], is one of the nonlinear analysis methods which has been widely used in both cardiovascular research and clinical applications. Here, we applied SampEn coupled with PR to prove whether the stages that we defined based on characteristics observed from the weekly PR models can be well clustered or not. SampEn was defined as the negative natural logarithm of the conditional probability that, within a data set of length $N$, two sequences similar to $m$ points (within a given tolerance $r$ ) will remain similar when the next point is included, without counting self-matches. It reduced the bias of ApEn and has closer agreement with theory for datasets with known probabilistic content. Lower value of SampEn indicates more self-similarity and regular time series (less complexity), whereas higher value indicates more irregularity (high complexity) and therefore is difficult to predict. SampEn can be calculated as follows.

For a given time series $X=x_{1}, x_{2}, \ldots, x_{N}, N$ is the total number of the data points. To form $m$-length subseries $X_{i m}$ within $X: X_{i m}=\left[x_{i}, x_{i+1}, \ldots, x_{i+m-1}\right]$, where $i=1,2, \ldots, N-$ $m+1$ and $m$ is the embedding dimension. Comparisons were then made against each $X_{i m}$ :

$$
B_{i}=\frac{n_{i}(m, r)}{N-m+1},
$$

where $B_{i}$ is the probability that any vectors will be similar to $X_{i m}$ and $n_{i}(m, r)$ is the number of vectors in $X_{j m}$ that are similar to $X_{i m}$ if and only if

$$
d\left(X_{i m}, X_{j m}\right) \leq r, \quad i \neq j,
$$

where $d\left(X_{i m}, X_{j m}\right)$ is defined as the maximal absolute difference between $X_{i m}$ and $X_{j m}$ and $r$ specifies the threshold for similarity (tolerance).

The total average probability can be calculated as

$$
B_{m}(r)=\frac{1}{N-m+1} \sum_{i=1}^{N-m+1} B_{i}(m, r) .
$$

$B_{m+1}(r)$ can be calculated in a similar process for an embedded dimension $m+1$.

Finally, SampEn, given $m$ and $r$, can be calculated by

$$
\operatorname{SampEn}(m, r)=\log \left[\frac{B_{m}(r)}{B_{m+1}(r)}\right] .
$$

To calculate SampEn, $m$ and $r$ are critical and may differ in different types of datasets. So far, no guidelines exist for 


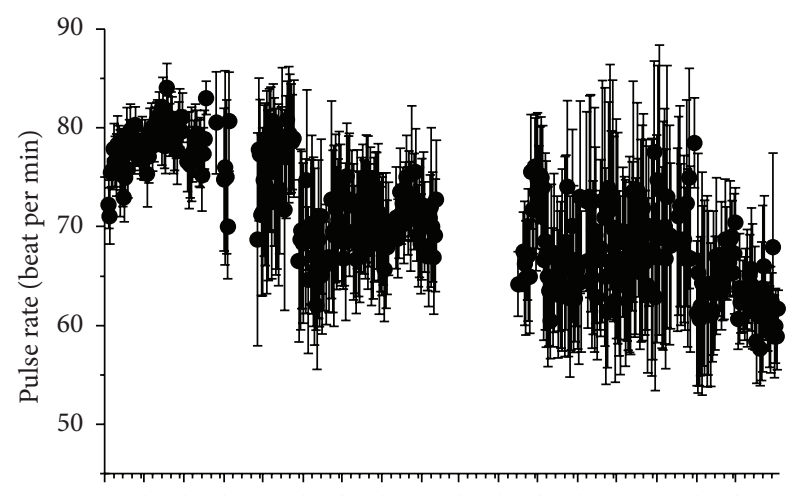

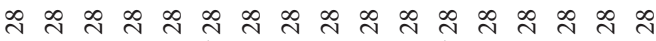

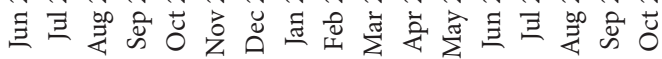

- Pregnant

(a)

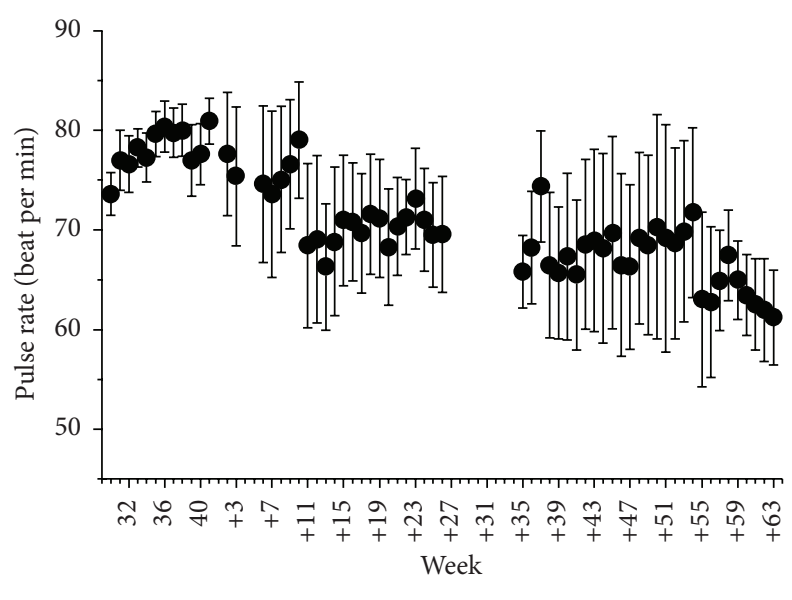

- Pregnant

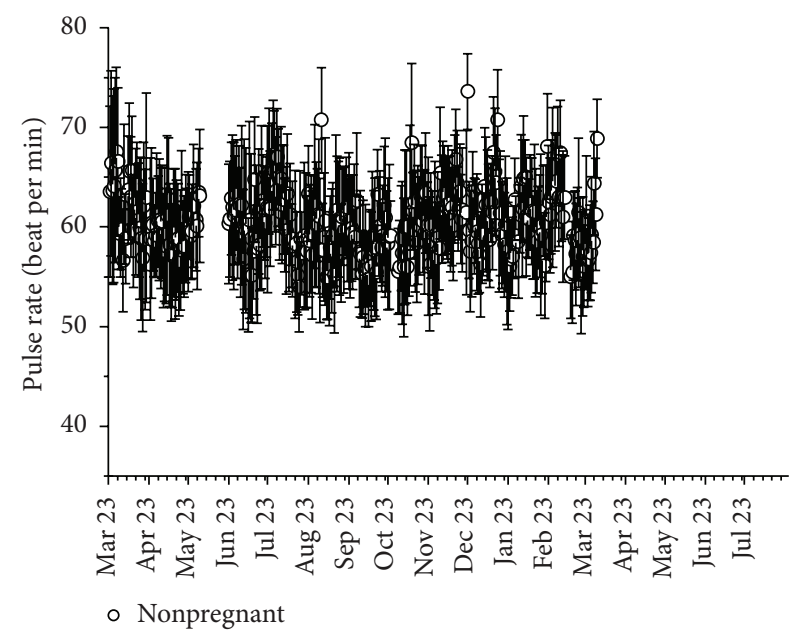

(b)

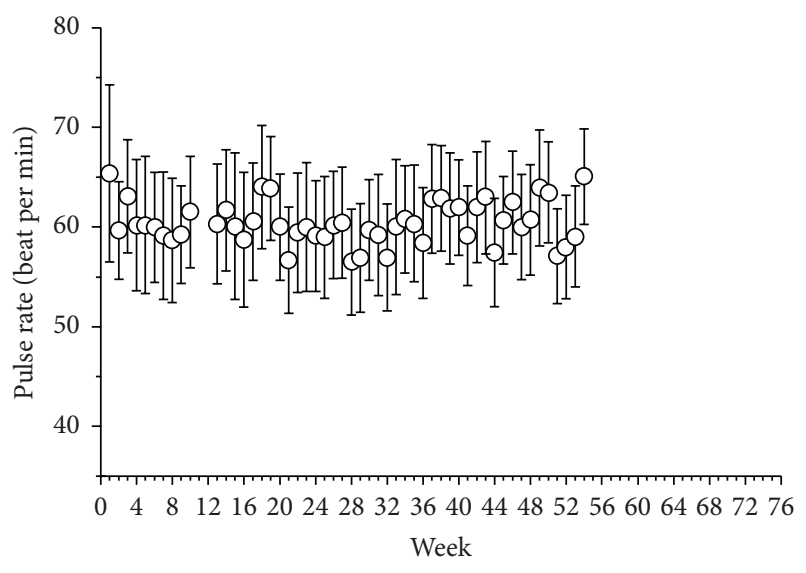

- Nonpregnant

(c)

(d)

FIGURE 3: Daily and weekly fluctuations of PR in both the pregnant subject ((a) and (c)) and the nonpregnant control individual ((b) and (d)). (a) and (b) show the daily variations, while (c) and (d) present weekly changes of PR throughout the entire recording period.

optimizing their values; recommended values are the use of $r$ between 0.1 and 0.25 and $m$ of 1 or 2 for data length $N$ ranging from 100 to 5,000 [27, 28].

\section{Results}

3.1. Daily and Weekly Pulse Rate Fluctuations. Daily mean $\mathrm{PR}$ and its SD were obtained from PR data series measured during one-night sleep. Weekly mean PR and its SD were calculated based on all data measured in the week. Figure 3 depicts the daily and weekly profiles of PR fluctuations of both subjects throughout the recording period. In the pregnant woman, daily PR changed in both its mean level and its SD throughout the whole period. The weekly mean PR $(74 \pm 2 \mathrm{bpm})$ increased from the 30th week of gestation and reached a peak $(80 \pm 3 \mathrm{bpm})$ on the $36 \mathrm{th}$ week. It then declined slightly during the 39th week $(77 \pm 4 \mathrm{bpm})$ at 2 weeks before childbirth. A sudden increase in PR mean level $(81 \pm 2 \mathrm{bpm})$ was observed on the day of delivery. It dropped within 6 weeks after delivery accompanied by a remarkable increase in the $\mathrm{SD}$ of the mean compared with that on the date of parturition. The PR raised in the following 4 weeks to $79 \pm 6 \mathrm{bpm}$ by the 10 th week. A sudden drop occurred in the 11th week postpartum to around $68 \mathrm{bpm}$. Thereafter, this declination slowed and the PR kept stable with only small variations over the following 44 weeks. There was a significant increase (by 9\%) in PR mean level on the 37 th week postpartum. Another notable decrease in the PR happened on the 55th week postpartum (by $12 \%$ compared with the previous week, to around $63 \mathrm{bpm}$ ), accompanied by a reduction in the SD. Conversely, both daily and weekly PR dynamics of the nonpregnant control subject remained stable throughout the recording period, with only small variations. On average, she had a mean PR of $60 \pm 6 \mathrm{bpm}$ with occasional higher PRs during sleep.

3.2. Weekly Pulse Rate Properties. To characterize the PR properties, weekly histograms and their skewness values for both subjects are plotted in Figures 4 and 5. In the healthy nonpregnant woman, the PR histogram presented 

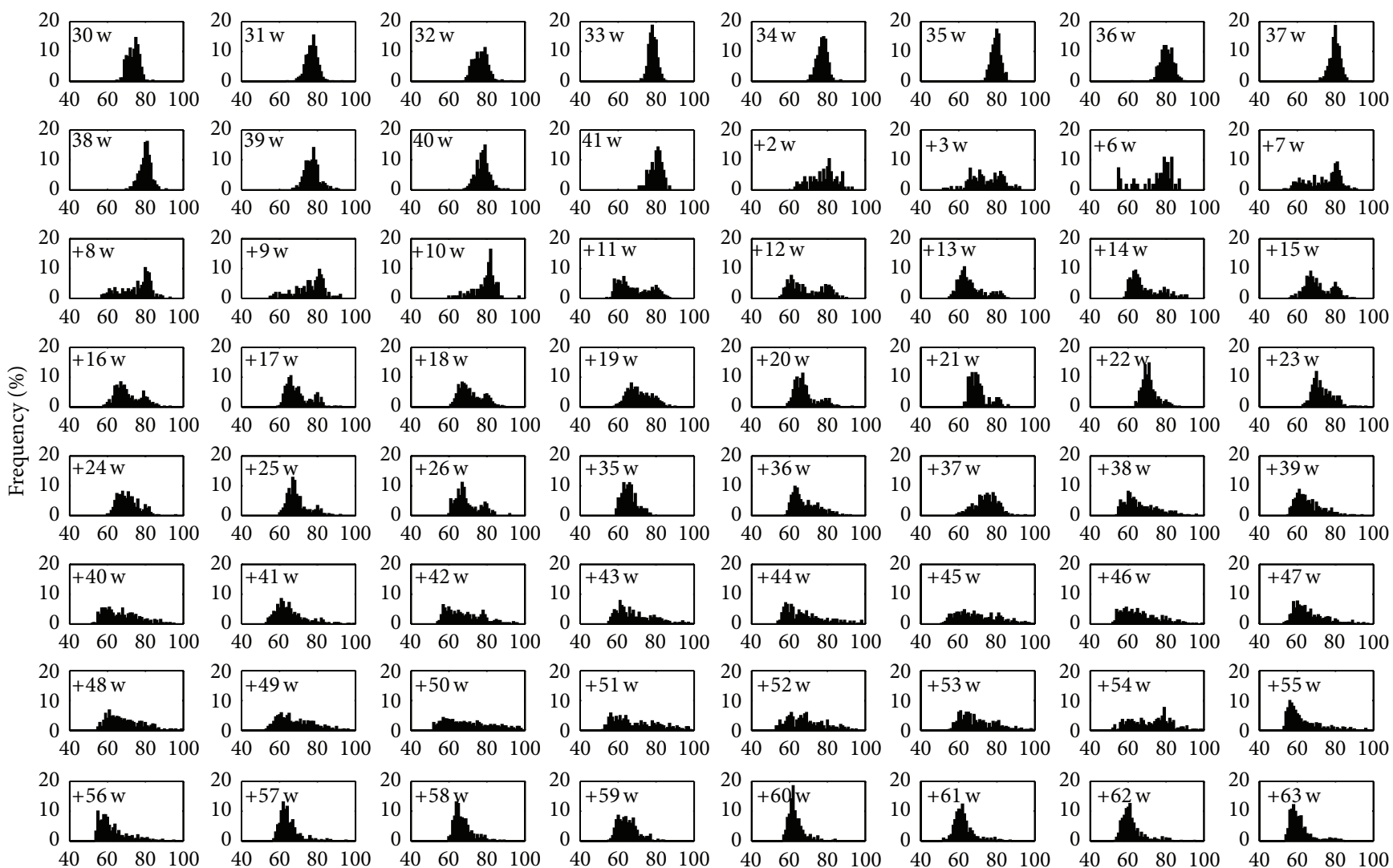

Pulse rate (beat per min)

(a)

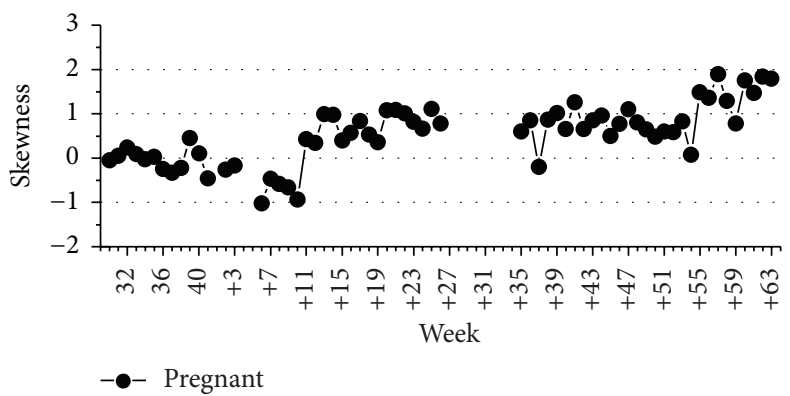

(b)

FIGURE 4: Characteristics of weekly PR distributions in the pregnant subject. (a) Weekly PR histograms, (b) weekly variations of the skewness index.

an approximately asymmetrical or a slightly positive skewed unimodal pattern as shown in Figure 5. In contrast, the pregnant woman demonstrated obvious weekly changes in the PR distribution with the progress of pregnancy (Figure 4).

In Figure 4, the data presented an approximately asymmetric but rather leptokurtic distribution from the 30th week indicating that most PRs were within a limited high level. The PR values quickly changed to wide and platykurtic distributions from the 2nd week after delivery indicating a severe increase in pulse rate variability. From the 2 nd to 10 th weeks postpartum, the PR presented a negatively skewed distribution (Figure 4(b)). In the 11th week postpartum, the PR distribution suddenly skewed to the right. A bimodal and positively skewed distribution was first detected within this period, indicating a transition point of the PR's development. This positive reversion of skewness suggested that the acceleration of PR was decreasing. The positively skewed distribution as well as the mean level of weekly PR waved around $70 \pm 2 \mathrm{bpm}$ from the 11 th week for the following 15 weeks. The weekly PR histograms in the 20th week changed from a wide platykurtic pattern back to a high and narrow distribution. This was during the transition time in which a previous study reported a restoration of the cardiovascular activities postpartum $[9,30]$. Indeed, the pattern of PR distribution after the 20th week was similar to that of the nonpregnant woman, as shown in Figure 4(b). However, the average PR 

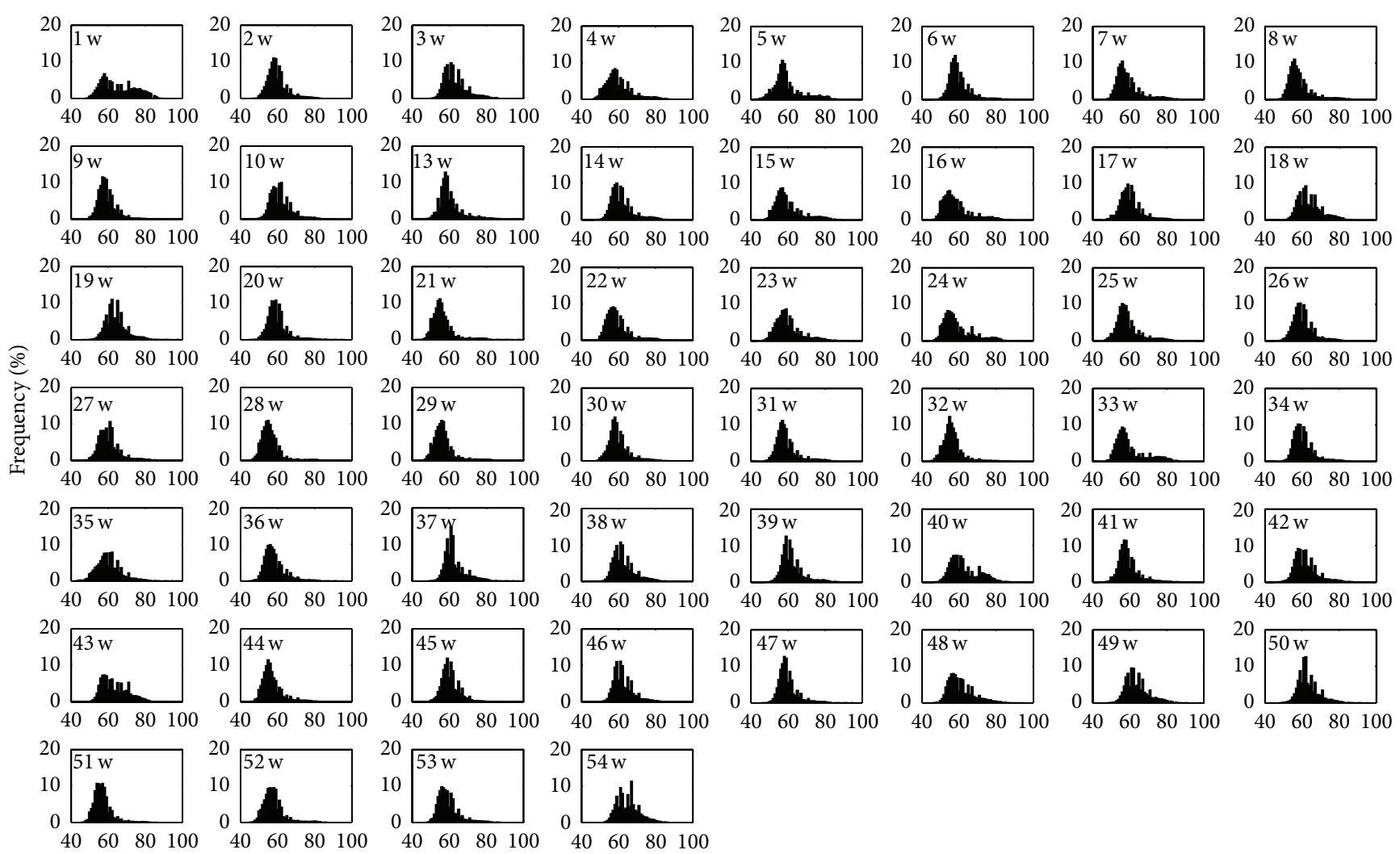

Pulse Rate (beat per min)

(a)

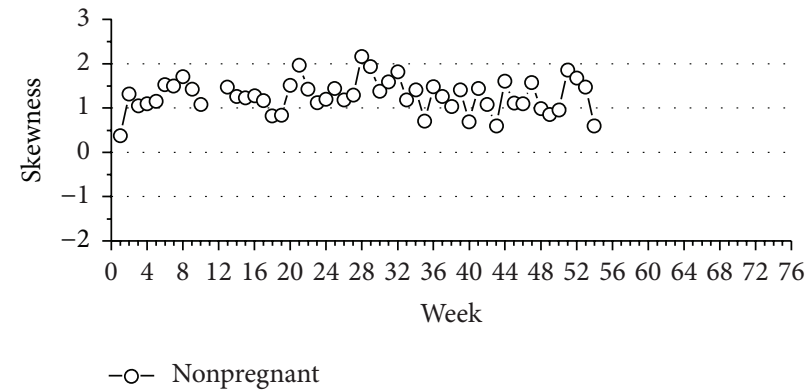

(b)

FIGURE 5: Characteristics of weekly PR distributions in the nonpregnant subject. (a) Weekly PR histograms, (b) weekly variations of the skewness index.

level was still high around $68 \mathrm{bpm}$ and the PR pattern was observed to remain unstable (Figure 4(a)). In other words, the recovery of cardiac function was still underway at the 20th week postpartum. This was confirmed by the 37th week, when the PR distribution gradually became wide and platykurtic again. This period lasted a long time until the 55th week postpartum, when there was a second significant reduction (from 72 to $63 \mathrm{bpm}$ ) in PR mean level. Meanwhile, the shape of the histogram changed from a wide-base, platykurtic pattern back to a leptokurtic pattern as shown in Figure 4(a). Most of the PR data declined to around $64 \mathrm{bpm}$ with only a few higher PRs creating skewness to the right. This transformation indicated a deceleration of the increased PR and both the pattern and PR level entered a stable state.
By this time, both the skewness and histogram pattern of PR distribution appear similar to that of the healthy nonpregnant woman, which may be inferred that the subject's PR dynamics had readjusted and recovered to a normal physical condition.

3.3. Weekly Indexes of the Gaussian Model. The weekly indexes of the fitted Gaussian model are shown in Figure 6. In the pregnant woman $(\mathrm{a}, \mathrm{c})$, the mean PRs of the two components were rather close to each other in the late trimester after the 30th week of gestation. A departure between the two peaks was detected after delivery. After the 6th week postpartum, the mean PR of the main component stayed high while the adjoining mean PR of the subcomponent headed 


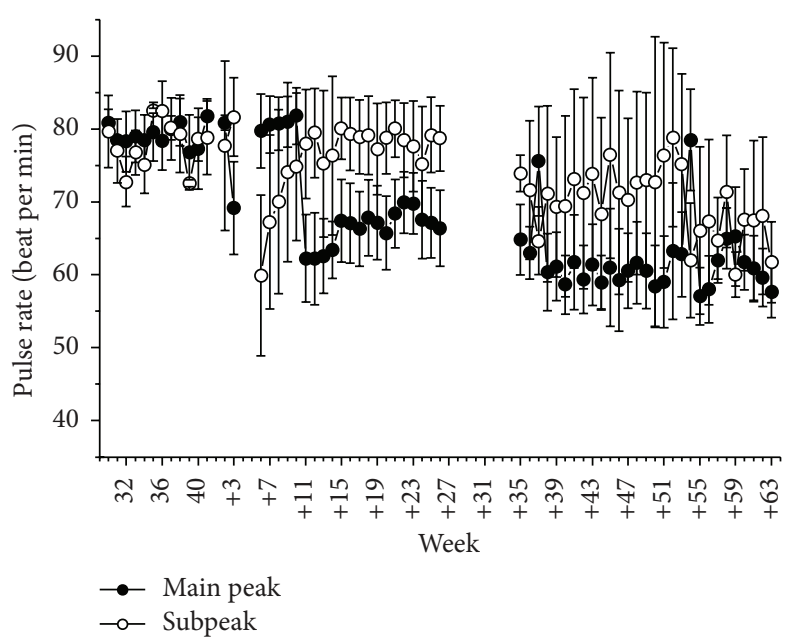

(a)

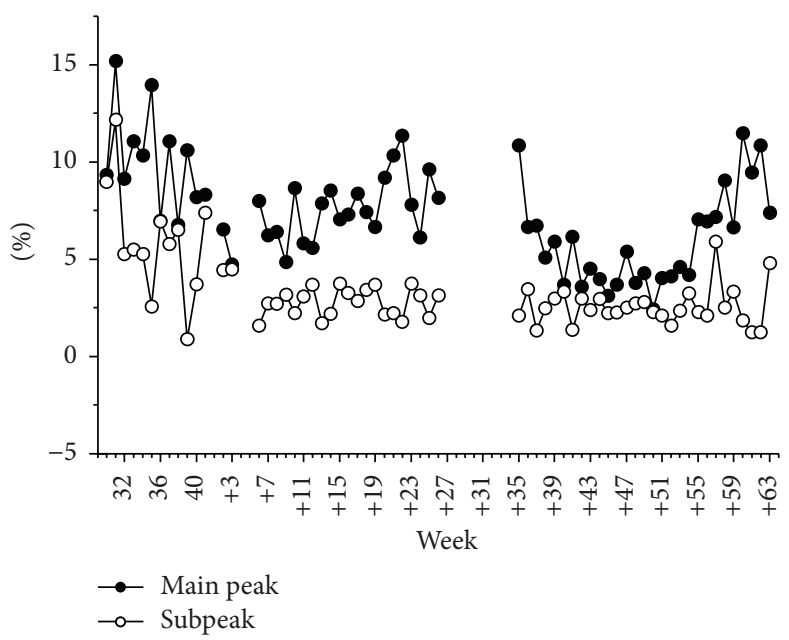

(c)

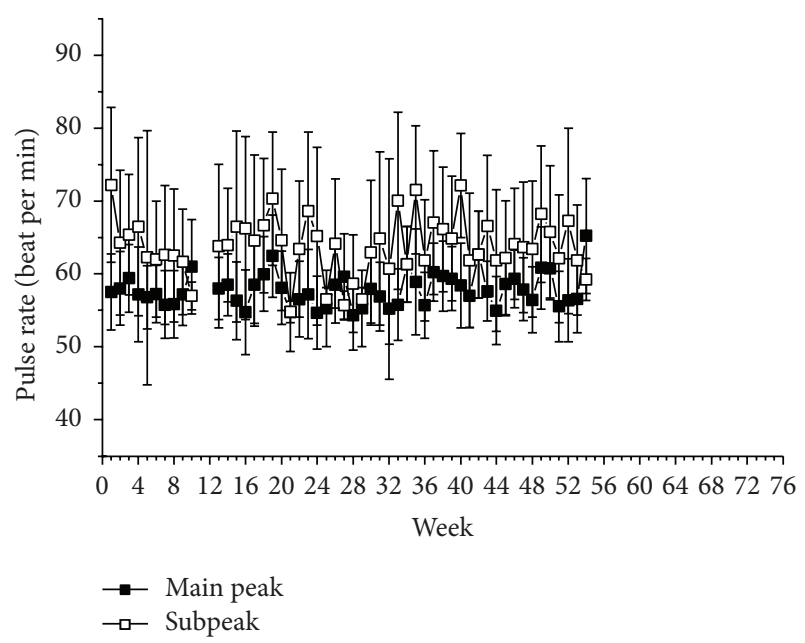

(b)

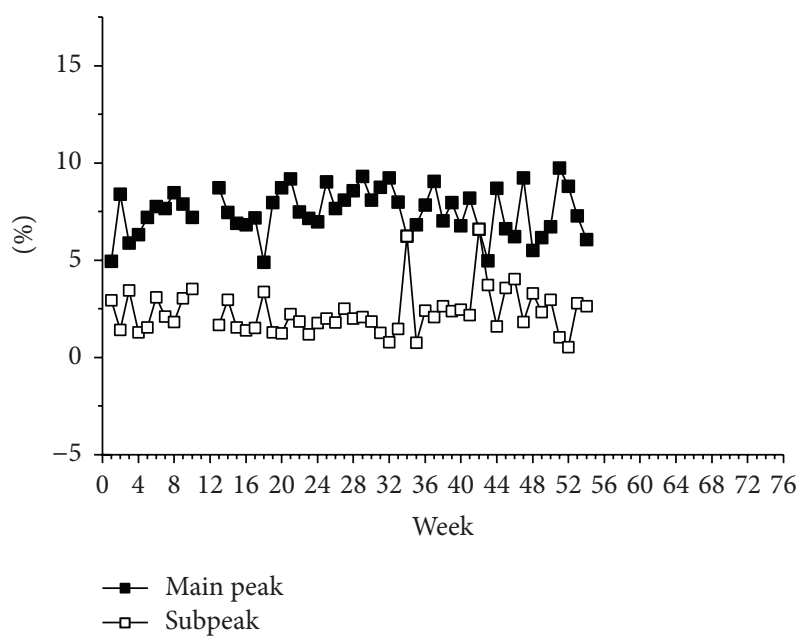

(d)

FIGURE 6: Changes in the two components of the weekly PR in both the pregnant ((a) and (c)) and nonpregnant ((b) and (d)) subjects. (a) and (b) show fluctuations of the mean PR values of the two components. (c) and (d) present variations in the respective percentage of each mean PR.

down to a significantly low level accompanied by an increase in the SD. A reversal of the two peaks took place on the subsequent 11th week, when the main mean PR dropped and left the mean PR of the subcomponent at a high level. The percentage of the main peak also increased. On the 37th week postpartum, the main mean PRs increased remarkably (by $12 \mathrm{bpm}$ ) accompanied by a decrease in the mean PR of the subcomponent (by $7 \mathrm{bpm}$ ) and a sharp reduction in the main peak percentage as shown in Figure 6(a). The percentages of both peaks approached each other at the 55th week after delivery and the main peak percentage rose back. Compared with that in the pregnant woman, the two components in the weekly PR data of the control subject appeared to be in a steady state throughout the whole period (Figures 6(b) and 6(d)). The mean PR of both components maintained a stable distance with each other and undulated at their own level (58 $\pm 5 \mathrm{bpm}$ versus $64 \pm 9 \mathrm{bpm})$. The percentages of the two peaks also remained stable with no apparent changes $(8 \pm 1 \%$ versus $2 \pm 1 \%)$.
3.4. Clustering the Stages. Based on above observations, we found some specific turning points during PR dynamic recovery, such as 6 th, 11 th, 20 th, 37 th, and 55 th weeks. Some of them have been reported in previous publications to indicate specific point of time when significant physiological changes take place, while others have not yet been revealed. We segmented the whole period recorded after delivery into six stages divided by the specific weeks as mentioned above (Table 1). A coupling of weekly SampEn and PR was used to approve our hypothesis of whether the six stages can be well clustered. Figures 7 and 8 show the weekly SampEn versus weekly PR extracted from pulse signal of the pregnant woman. It can be observed that the distributions of weekly SampEn and PR representing different stages are presented in much more compact and well-defined clusters when using the main (Figure 8(a)) or subpeaks (Figure 8(b)) of weekly PRs than using only the mean PRs (Figure 7). Centers of each cluster are listed in Table 2. It further proves that the recovery of cardiac functions underwent different stages, and the use 
TABLE 1: Segmentation of recorded data and main features of each stage.

\begin{tabular}{|c|c|c|c|c|c|}
\hline Stage & Period (week) & $\begin{array}{c}\text { PR (bpm) } \\
(\text { mean } \pm S D)\end{array}$ & Histogram & $\begin{array}{c}\text { Two peaks of } \\
\text { Gaussian model }\end{array}$ & $\begin{array}{c}\text { SampEn (mean } \pm \\
\text { SD) }\end{array}$ \\
\hline $\mathrm{AP}^{\mathrm{a}}$ & 30th 41th & $78 \pm 2$ & Asymmetric, leptokurtic & Similar High & $3.15 \pm 0.17$ \\
\hline $\mathrm{PP}^{\mathrm{b}} 1$ & +1 th $\sim+5$ th & $77 \pm 2$ & Negatively skewed, platykurtic & Similar High & $3.35 \pm 0.22$ \\
\hline PP 2 & +6 th $\sim 10$ th & $76 \pm 2$ & Negatively skewed, platykurtic & $\begin{array}{l}\text { Main peak stays } \\
\text { high; subpeak } \\
\text { drops down }\end{array}$ & $3.96 \pm 0.03$ \\
\hline PP 3 & +11 th $\sim+19$ th & $70 \pm 2$ & Positively skewed, platykurtic, and bimodal & $\begin{array}{l}\text { Main peak } \\
\text { drops down; } \\
\text { subpeak } \\
\text { reverses high }\end{array}$ & $3.63 \pm 0.07$ \\
\hline PP 4 & +20 th $\sim 36$ th & $70 \pm 2$ & Positively skewed, leptokurtic & $\begin{array}{l}\text { Main peak stays } \\
\text { low; subpeak } \\
\text { stays high }\end{array}$ & $3.43 \pm 0.09$ \\
\hline PP 5 & +37 th $\sim+54$ th & $69 \pm 2$ & Positively skewed, platykurtic & Both decrease & $3.62 \pm 0.08$ \\
\hline PP 6 & +55 th $\sim+63 \mathrm{rd}$ & $64 \pm 2$ & Positively skewed, leptokurtic & $\begin{array}{l}\text { Both come close } \\
\text { together }\end{array}$ & $3.30 \pm 0.08$ \\
\hline $\begin{array}{l}\text { Nonpregnant } \\
\text { woman }\end{array}$ & Whole period & $60 \pm 2$ & Positively skewed, leptokurtic & $\begin{array}{l}\text { Both stay close } \\
\text { together }\end{array}$ & $2.40 \pm 0.20$ \\
\hline
\end{tabular}

“+” indicates weeks after delivery.

a Antepartum.

${ }^{\mathrm{b}}$ Postpartum.

TABLE 2: The center of each cluster.

\begin{tabular}{lccc}
\hline Stage & SampEn versus weekly mean PR & $\begin{array}{c}\text { SampEn versus main peak of } \\
\text { weekly PR model }\end{array}$ & $\begin{array}{c}\text { SampEn versus } \\
\text { subpeak of weekly PR } \\
\text { model }\end{array}$ \\
\hline AP $^{\mathrm{a}}$ & $(3.15$, and 74) & $(3.15$, and 79) & $(3.15$, and 78) \\
PP 1 & $(3.35$, and 77) & $(3.35$, and 75) & $(3.35$, and 80) \\
PP 2 & $(3.96$, and 76) & $(3.96$, and 81) & $(3.96$, and 75) \\
PP 3 & $(3.63$, and 70) & $(3.63$, and 65) & $(3.43$, and 77) \\
PP 4 & $(3.43$, and 70) & $(3.43$, and 67) & $(3.62$, and 72) \\
PP 5 & $(3.62$, and 69) & $(3.62$, and 62) & $(3.30$, and 66) \\
PP 6 & $(3.30$, and 64) & $(3.30$, and 61) & \\
\hline
\end{tabular}

${ }^{\mathrm{a}}$ Antepartum.

${ }^{\mathrm{b}}$ Postpartum.

of Gaussian model can better reveal the characteristics of the under dynamics of PR development.

\section{Discussion}

Late pregnancy is associated with dramatic cardiovascular adaptations. Increased cardiac output, hormonal changes, increased circulation burden, and aortocaval compression as a result of the gravid uterus are responsible for an increased PR when supine and right lateral decubitus positions are assumed during sleep [29]. The rise in cardiac output in late pregnancy is mostly caused by a rise in the HR for maintaining an elevated cardiac output as term approaches $[6,7,30-32]$. We observed that the mean PR increased after the 31st week during pregnancy compared with the 30th week. This significant rise might have been because of the fetus's weight gain after the 31st week. This could increase aortocaval compression as well as the circulation burden. Another possible reason could be the rise in plasma volume through increased aldosterone levels. The PR rose apparently during the late phase of the last trimester but began to decrease 2 weeks before term. This declination was in accordance with previous reports that the HR decreases slightly in the final weeks of pregnancy [2]. Greater aortocaval compression was expected to interfere with autonomic nervous activity with induced higher sympathetic activation and greater vagal suppression. Therefore, the relief from aortocaval compression after delivery might have contributed to an increase in HRV.

The PR declined shortly after delivery and reached a relatively low level. This was in accordance with a report that most of the decrease, including cardiac output and HR, occurs within 2 weeks after delivery [2, 6, 7, 9]. However, the PR of our subject did not return to normal within 6 weeks, as asserted in previous studies [1, 2, 6, 9-11]. Conversely, it increased from the 7 th week to the 11th week postpartum. SampEn also increased significantly during this 


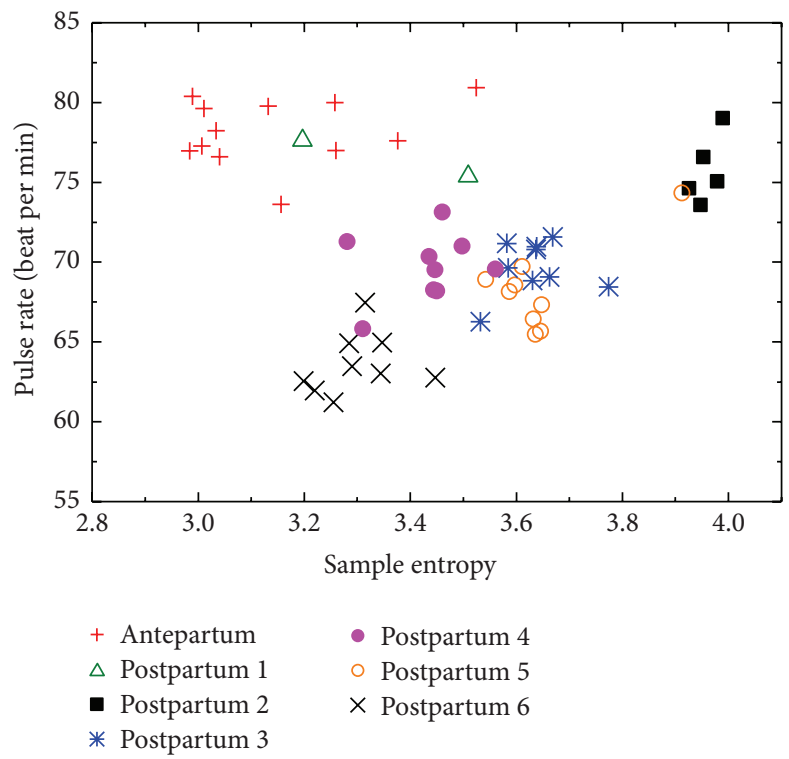

FIGURE 7: Plot of weekly PRs versus weekly SampEn features of different stages before and after delivery.

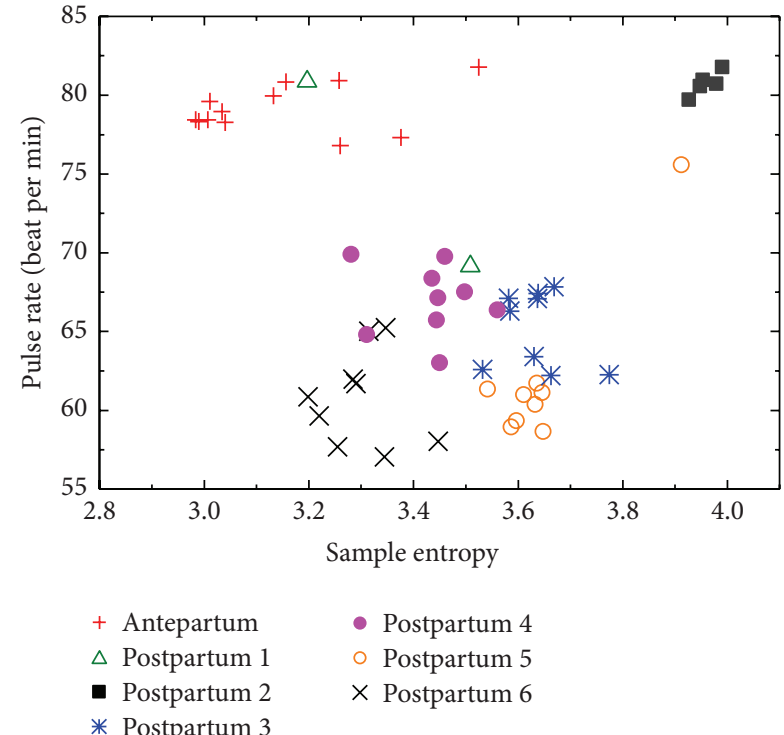

(a)

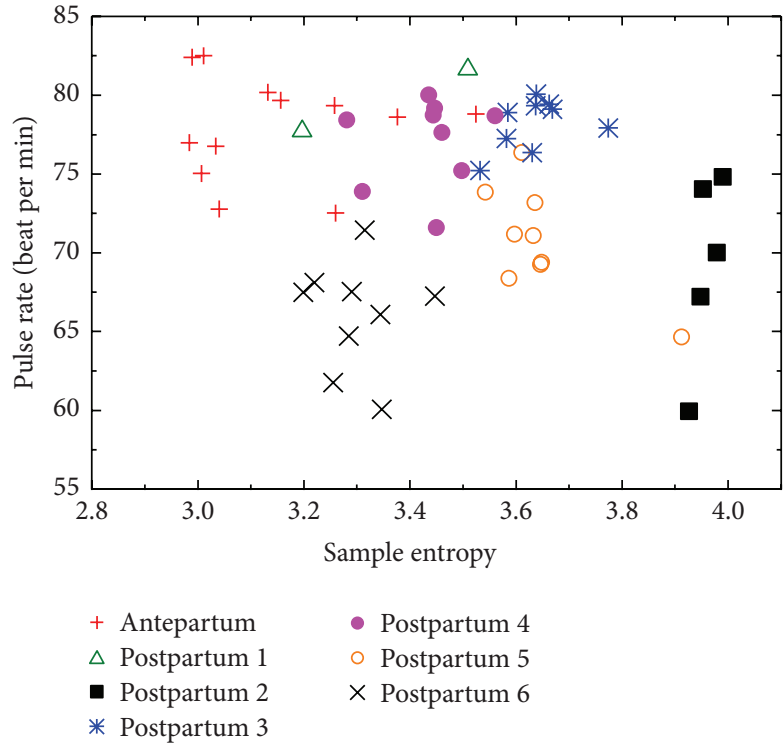

(b)

FIGURE 8: Plots of main peaks of weekly PRs versus weekly SampEn features (a) as well as subpeaks of weekly PRs versus weekly SampEn features (b) of different stages during before and after delivery.

stage (Figures 7 and 8) indicating an increase in HR nonlinearity. Possible reasons for this rebound might be that although the circulation burden was released postpartum, the subject's body still needed time to readjust to a new physiological state and to recover from postpartum exhaustion, uterine contraction, "after-pains," perineal pain, and general edema. In one report, the recovery period was around 20 weeks $[7,9]$. However, other studies indicated that cardiac activities might not return to original levels even after 1 year $[8,11]$.

Previous studies reported different times or even an indefinite time for recovery by recording heart rate changes. Possible reasons might be due to different data collection and analysis methods. Most of previous conclusions were based on data collected from regular physical examinations at intermittently rather than on a daily continuous base. Therefore, it is difficult to obtain a complete profile. In our study, the sleep monitoring system enabled a longitudinal study over a long period. Compared with conventional measurements, it seemed to be more effective in monitoring PR dynamics over time. In addition, signals were recorded during the restful state of nighttime sleep periods while the subject was unconscious, which could avoid interference from the outside environment and the subject's daily activities. Therefore, it can better reflect the underlying biorhythms. This similar 
protocol had been proved to success in detection of health changes in a patient with cardiac disorder in our previous research [24].

HR and HRV are two of the most important parameters in evaluating cardiac function. Previous studies usually calculated HR or HRV within limited recording period. Even the averaged HR have dropped to a reference baseline, the cardiac system may be still not fully recovered. A combined use of histogram and a two-Gaussian mixture model can visually reveal the dynamic transition of PR by decomposing weekly PR into two components. In Figure 7, stages 3 and 4 during postpartum period cannot be classified well when using only the weekly mean PR. But after decomposing them into two components, stages can be clustered and distinguished better with each other as shown in Figure 8. Thus, the introduced model enables a better capture of the characteristics in adaptations and the restorations process of the cardiac function during normal pregnancy. We have summarized the main features of different stages in Table 1.

Although only one pregnant woman was included in the current study, it is worth noting that our observations are in quite accordance with some previous reports regarding the time point for significant PR changes during pregnancy and after delivery [2, 6, 7]. Particularly, one study [8] depicted a cardiovascular data profile before, during, and 52 weeks after normal pregnancy based on intermittent collected data from 30 women. The profile was quite similar with our results obtained from daily measurement of a single subject. However, whether the PR dynamics return to a preconception level cannot be concluded. We still need the prepregnant or early pregnant data for reference. Possible time and stages required for recovery have been also suggested by our study; even though conclusive results cannot be given before more subjects can be included, at least it can be derived from our findings that recovery from pregnancy may take longer time than previously reported 6 or 20 weeks.

\section{Conclusions}

We have introduced a feasible approach to characterize daily PR dynamics during pregnancy and after delivery. In this study, the PR did not decline steadily after delivery but differed in stages. Relief of circulation burden and aortocaval compression as a result of delivery might be the main cause of a significant return to normal PR values during an early stage after delivery. Postpartum changes in hormones-including readjustments of aldosterone, estrogen, and progesterone levels-might also contribute to the return to normal cardiac activity after delivery. However, the later stages of recovery seemed to require a much longer period. In our study, the pregnant woman underwent five stages in recovery and it took about one year for the PR dynamics to readjust to a normal physical condition. We hope the findings can provide helpful applications in maternal cardiac monitoring and studying the maternal cardiac progression during pregnancy and after delivery.

\section{Conflict of Interests}

The authors declare that there is no conflict of interests regarding the publication of this paper.

\section{Acknowledgments}

The authors would like to thank the volunteers for their endurance in daily data collection and colleagues in the research project. The support provided in part by the University of Aizu Competitive Research Funding P-21 is gratefully acknowledged.

\section{References}

[1] S. Hunter and S. C. Robson, "Adaptation of the maternal heart in pregnancy," British Heart Journal, vol. 68, no. 12, pp. 540-543, 1992.

[2] J. J. Duvekot and L. L. H. Peeters, "Maternal cardiovascular hemodynamic adaptation to pregnancy," Obstetrical \& Gynecological Survey, vol. 49, no. 12, pp. S1-S14, 1994.

[3] T. L. Weissgerber and L. A. Wolfe, "Physiological adaptation in early human pregnancy: adaptation to balance maternal-fetal demands," Applied Physiology, Nutrition and Metabolism, vol. 31, no. 1, pp. 1-11, 2006.

[4] O. Savu, R. Jurcuţ, S. Giuşcă et al., "Morphological and functional adaptation of the maternal heart during pregnancy," Circulation: Cardiovascular Imaging, vol. 5, no. 3, pp. 289-297, 2012.

[5] K. Melchiorre, R. Sharma, and B. Thilaganathan, "Cardiac structure and function in normal pregnancy," Current Opinion in Obstetrics and Gynecology, vol. 24, no. 6, pp. 413-421, 2012.

[6] A. C. C. van Oppen, R. H. Stigter, and H. W. Bruinse, "Cardiac output in normal pregnancy: a critical review," Obstetrics \& Gynecology, vol. 87, no. 2 I, pp. 310-318, 1996.

[7] S. Fujitani and M. R. Baldisseri, "Hemodynamic assessment in a pregnant and peripartum patient," Critical Care Medicine, vol. 33, no. 10, pp. S354-S361, 2005.

[8] J. F. Clapp III and E. Capeless, "Cardiovascular function before, during, and after the first and subsequent pregnancies," The American Journal of Cardiology, vol. 80, no. 11, pp. 1469-1473, 1997.

[9] S. C. Robson, W. Dunlop, M. Moore, and S. Hunter, "Haemodynamic changes during the puerperium: a Doppler and Mmode echocardiographic study," British Journal of Obstetrics and Gynaecology, vol. 94, no. 11, pp. 1028-1039, 1987.

[10] W. C. Mabie, T. G. DiSessa, L. G. Crocker, B. M. Sibai, and K. L. Arheart, "A longitudinal study of cardiac output in normal human pregnancy," American Journal of Obstetrics \& Gynecology, vol. 170, no. 3, pp. 849-856, 1994.

[11] E. L. Capeless and J. F. Clapp, "When do cardiovascular parameters return to their preconception values?" American Journal of Obstetrics \& Gynecology, vol. 165, no. 4, pp. 883-886, 1991.

[12] N. Kudo, H. Shinohara, and H. Kodama, "Heart rate variability biofeedback intervention for reduction of psychological stress during the early postpartum period," Applied Psychophysiology Biofeedback, vol. 39, no. 3-4, pp. 203-211, 2014.

[13] A. Shimizu, H. Nishiumi, Y. Okumura, and K. Watanabe, "Depressive symptoms and changes in physiological and social 
factors 1 week to 4 months postpartum in Japan," Journal of Affective Disorders, vol. 179, pp. 175-182, 2015.

[14] F. T. Burgut, A. Bener, S. Ghuloum, and J. Sheikh, "A study of postpartum depression and maternal risk factors in Qatar," Journal of Psychosomatic Obstetrics and Gynecology, vol. 34, no. 2, pp. 90-97, 2013.

[15] N. Sohnchen, K. Melzer, B. M. Tejada et al., "Maternal heart rate changes during labour," European Journal of Obstetrics \& Gynecology and Reproductive Biology, vol. 158, pp. 173-178, 2011.

[16] D. J. Sherman, E. Frenkel, Y. Kurzweil, A. Padua, S. Arieli, and M. Bahar, "Characteristics of maternal heart rate patterns during labor and delivery," Obstetrics \& Gynecology, vol. 99, no. 4, pp. 542-547, 2002.

[17] S. C. Robson, R. J. Boys, S. Hunter, and W. Dunlop, "Maternal hemodynamics after normal delivery and delivery complicated by postpartum hemorrhage," Obstetrics and Gynecology, vol. 74, no. 2, pp. 234-239, 1989.

[18] P. Ray, G. J. Murphy, and L. E. Shutt, "Recognition and management of maternal cardiac disease in pregnancy," British Journal of Anaesthesia, vol. 93, no. 3, pp. 428-439, 2004.

[19] A. Wacker-Gußmann, M. Thriemer, M. Yigitbasi, F. Berger, and N. Nagdyman, "Women with congenital heart disease: longterm outcomes after pregnancy," Clinical Research in Cardiology, vol. 102, no. 3, pp. 215-222, 2013.

[20] C. V. Russoniello, V. Pougtachev, E. Zhirnov, and M. T. Mahar, "A measurement of electrocardiography and photoplethesmography in obese children," Applied Psychophysiology Biofeedback, vol. 35, no. 3, pp. 257-259, 2010.

[21] F. Javed, P. M. Middleton, P. Malouf et al., "Frequency spectrum analysis of finger photoplethysmographic waveform variability during haemodialysis," Physiological Measurement, vol. 31, no. 9, pp. 1203-1216, 2010.

[22] G. S. H. Chan, A. Fazalbhoy, I. Birznieks, V. G. Macefield, P. M. Middleton, and N. H. Lovell, "Spontaneous fluctuations in the peripheral photoplethysmographic waveform: roles of arterial pressure and muscle sympathetic nerve activity," The American Journal of Physiology-Heart and Circulatory Physiology, vol. 302, no. 3, pp. H826-H836, 2012.

[23] J. Allen and A. Murray, "Age-related changes in the characteristics of the photoplethysmographic pulse shape at various body sites," Physiological Measurement, vol. 24, no. 2, pp. 297-307, 2003.

[24] Y. Chen and W. Chen, "Long-term tracking of a patient's health condition based on pulse rate dynamics during sleep," Annals of Biomedical Engineering, vol. 39, no. 12, pp. 2922-2934, 2011.

[25] W. Chen, X. Zhu, and T. Nemoto, "A new sensory device and optimal position for monitoring HR/RR during sleep," in World Congress on Medical Physics and Biomedical Engineering, September 7-12, 2009, Munich, Germany, vol. 25/7 of IFMBE Proceedings, pp. 126-129, Springer, Berlin, Germany, 2009.

[26] X. Zhu, W. Chen, T. Nemoto et al., "Real-time monitoring of respiration rhythm and pulse rate during sleep," IEEE Transactions on Biomedical Engineering, vol. 53, no. 12, pp. 25532563, 2006.

[27] S. M. Pincus, "Approximate entropy as a measure of system complexity," Proceedings of the National Academy of Sciences of the United States of America, vol. 88, no. 6, pp. 2297-2301, 1991.

[28] J. S. Richman and J. R. Moorman, "Physiological time-series analysis using approximate and sample entropy," The American Journal of Physiology-Heart and Circulatory Physiology, vol. 278, no. 6, pp. H2039-H2049, 2000.
[29] G.-Y. Chen, C.-D. Kuo, M.-J. Yang, H.-M. Lo, and Y.-S. Tsai, "Return of autonomic nervous activity after delivery: role of aortocaval compression," British Journal of Anaesthesia, vol. 82, no. 6, pp. 932-934, 1999.

[30] S. C. Robson, W. Dunlop, R. J. Boys, and S. Hunter, "Cardiac output during labour," British Medical Journal, vol. 295, no. 6607, pp. 1169-1172, 1987.

[31] F. Westling, I. Milsom, H. Zetterstrom, and B. Ekstrom-Jodal, "Effects of nitrous oxide/oxygen inhalation on the maternal circulation during vaginal delivery", Acta Anaesthesiologica Scandinavica, vol. 36, no. 2, pp. 175-181, 1992.

[32] J. G. Ouzounian and U. Elkayam, "Physiologic changes during normal pregnancy and delivery," Cardiology Clinics, vol. 30, no. 3, pp. 317-329, 2012. 


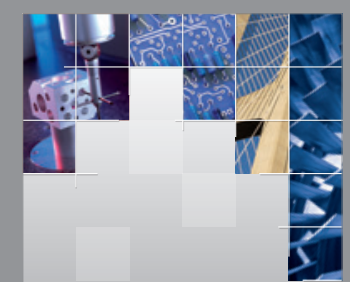

\section{Enfincering}
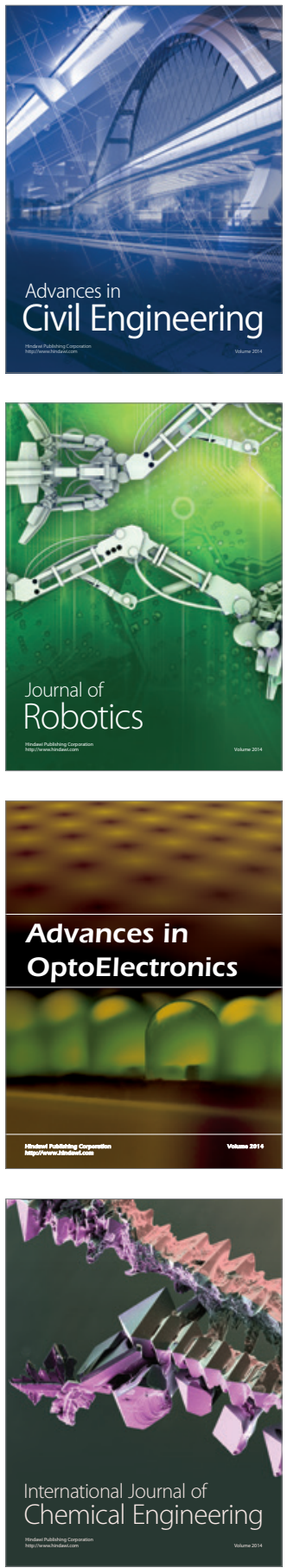

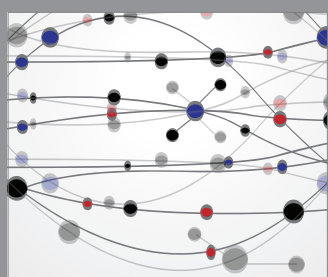

The Scientific World Journal

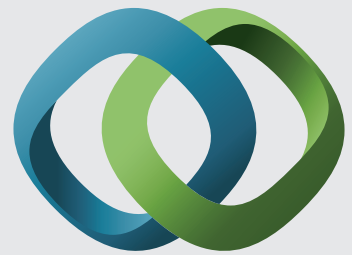

\section{Hindawi}

Submit your manuscripts at

http://www.hindawi.com
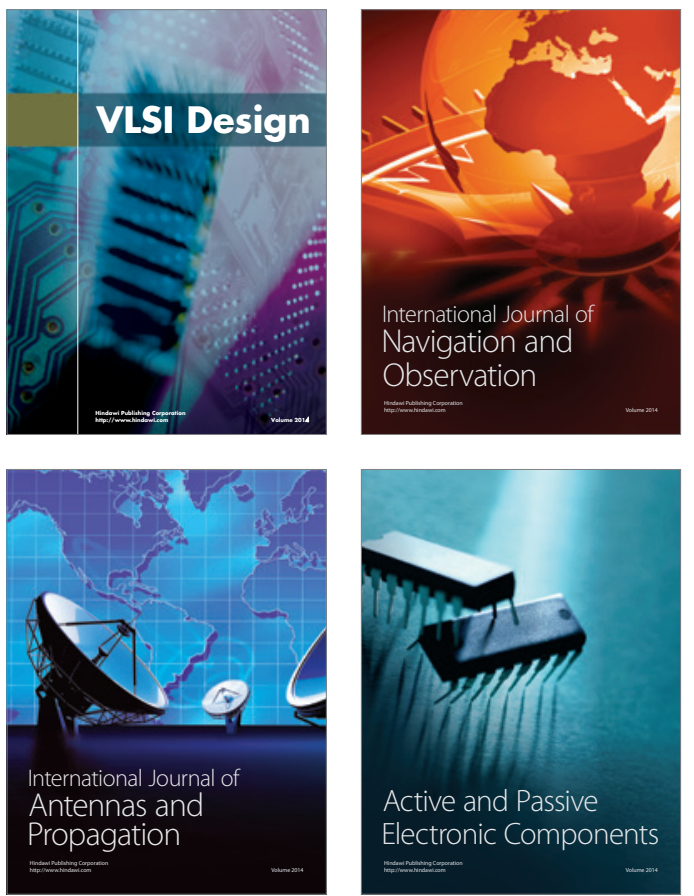
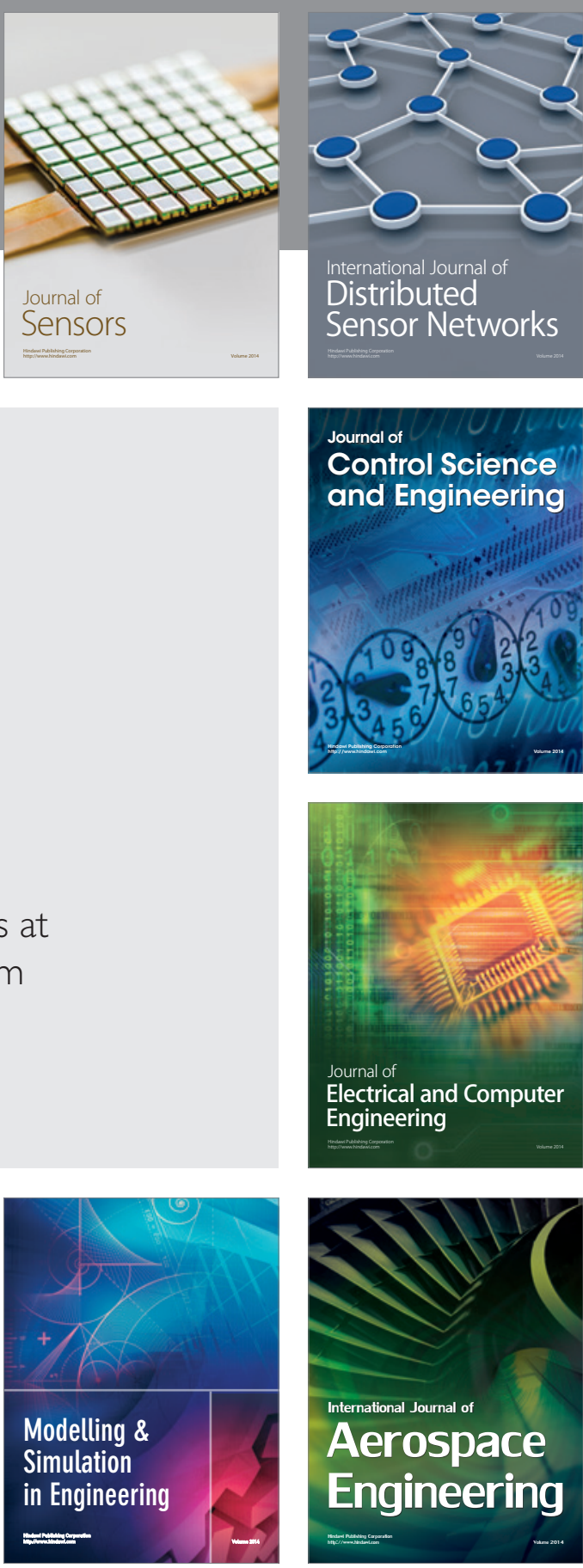

International Journal of

Distributed

Sensor Networks

Journal of

Control Science

and Engineering
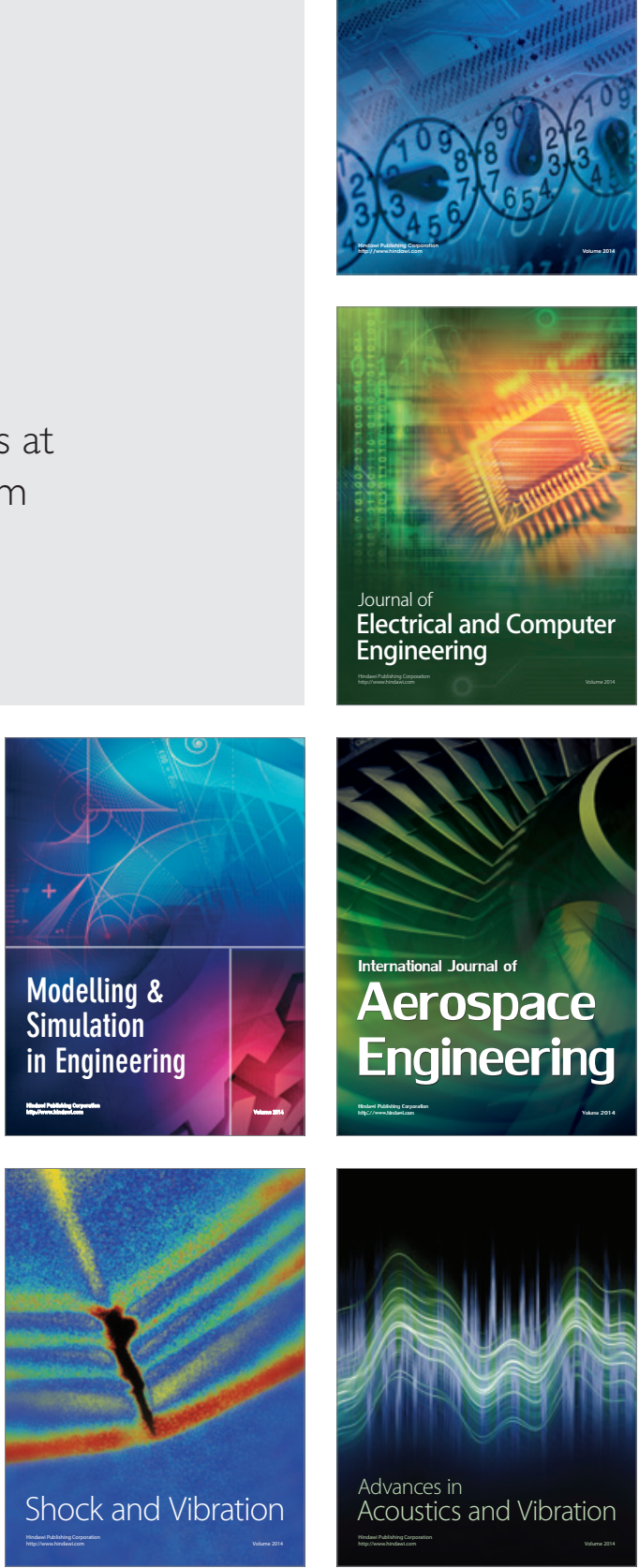